\title{
Africa's critical care capacity before COVID-19
}

\author{
ET Ayebale,' (iD NJ Kassebaum, ${ }^{2}$ iD AM Roche, ${ }^{3}$ iD BM Biccard ${ }^{4}$ iD \\ ${ }^{1}$ University of Washington, Department of Anesthesiology and Pain Medicine, Harborview Medical Center, United States of America \\ ${ }^{2}$ University of Washington, Institute for Health Metrics and Evaluation, United States of America \\ ${ }^{3}$ University of Washington, Department of Anesthesiology and Pain Medicine, Harborview Medical Center, United States of America \\ ${ }^{4}$ Department of Anaesthesia and Perioperative Medicine, University of Cape Town and Groote Schuur Hospital, South Africa \\ Corresponding author, email: eayebale@uw.edu
}

Critical care capabilities in affluent countries have been overwhelmed by the 2019 novel coronavirus disease (COVID-19) pandemic. Data from the African Surgical Outcomes Study (ASOS) ${ }^{1}$ suggests that this critical care crisis will be significantly worse in Africa.

Data from ASOS, and three country audits (South Africa [Alex van den Heever, unpublished data], Kenya ${ }^{2}$ and Uganda $^{3}$ ) were used to determine the critical care bed capacity for Africa. Using Global Burden of Disease (GBD) 2017 population estimates, and World Bank estimates of hospital beds per capita, we estimate 1055846 hospital beds and 10784 critical care beds across Africa for 1.2 billion people, equating to $0.8(0.3-1.45)$ critical care beds per 100000 population (analysis and individual country data shown in Supplementary material, and the dataset can be found at http://dx.doi.org/10.17632/crsm4s6dc4.1). Only six countries have a median of more than two critical care beds per 100000 population (Figure 1). It is likely that the true number of critical care beds in most African countries is lower than we have calculated, as the majority of hospitals in ASOS were secondary and tertiary hospitals. ' In contrast, Europe has 14 times the critical care bed capacity at 11.5 beds per 100000 population, ${ }^{4}$ and has still had to provide extra critical care beds outside of intensive care units (ICUs).

The insufficient critical care beds will be further compromised by a limited healthcare workforce in Africa. There are insufficient critical care nurses. Unpublished data from ASOS show that over $50 \%$ of participating ICUs were unable to maintain a nurse: patient ratio of at least 1:2. Anaesthetists comprise a substantial proportion of providers capable of providing critical care support and ventilator management. Yet, the World Federation of Societies of Anaesthesiologists (WFSA) ${ }^{5}$ estimates that $78 \%$ of African countries have non-physician anaesthetists comprising more than $50 \%$ of their anaesthesia workforce. Reassigning anaesthesia providers to cover this gap in the critical care workforce as has been done elsewhere would not be possible in Africa due to insufficient anaesthesia providers (Figure 1). There is little data on other crucial components of critical care in Africa, including the actual number of functional ventilators and patient monitors, access to medical oxygen, airway, resuscitation, and personal protective equipment, and ICU drugs.

With this potential critical care crisis in the COVID-19 pandemic in Africa, a mass educational programme on basic management of critically ill patients in a resource-limited setting for noncritical care staff is urgently needed to minimise unnecessary, excess mortality across Africa.

\section{Authors' contributions}

All authors equally contributed to the literature search, figures, study design, data collection, data analysis, data interpretation, writing, and approval of the final manuscript.

\section{Role of the funding source}

No funding sources to disclose. The corresponding author, $\mathrm{Dr}$ Emmanuel Timarwa Ayebale, had full access to all the data in the study and had final responsibility for the decision to submit for publication.

\section{Conflict of interest}

All authors have nothing to disclose.

$\Delta$

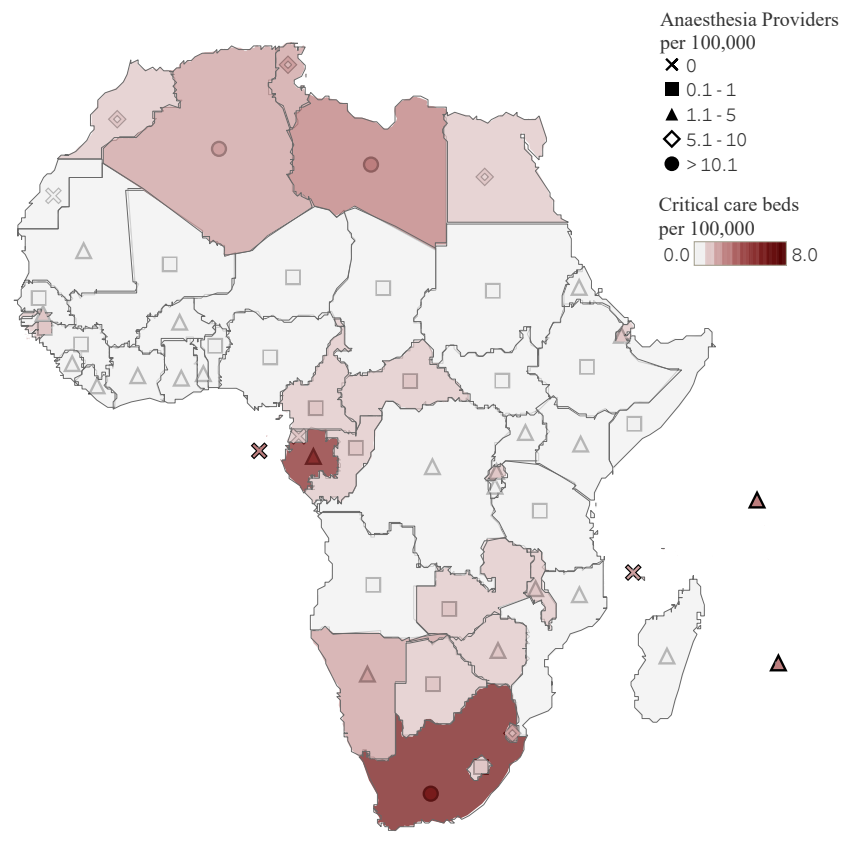




\section{ORCID}

ET Ayebale (iD) https://orcid.org/0000-0001-8744-3960 NJ Kassebaum (iD) https://orcid.org/0000-0002-4299-9348 AM Roche (iD) https://orcid.org/0000-0002-4802-4330 BM Biccard (iD) http://orcid.org/0000-0001-5872-8369

\section{References}

1. Biccard BM, Madiba TE, Kluyts H-L, et al. Perioperative patient outcomes in the African Surgical Outcomes Study: a 7-day prospective observational cohort study. The Lancet. 2018;391(10130):1589-98. https://doi.org/10.1016/ S0140-6736(18)30001-1.
2. Okech UK, Chokwe T, Mung'ayi V. The operational setup of intensive care units in a low-income country in East Africa. East African Medical Journal. 2015;92(2):72-80

3. Atumanya $P$, Sendagire $C$, Wabule $A$, et al. Assessment of the current capacity of intensive care units in Uganda; A descriptive study. Journal of Critical Care. 2020;55:95-99. https://doi.org/10.1016/j.jcrc.2019.10.019.

4. Rhodes $A$, Ferdinande $P$, Flaatten $H$, et al. The variability of critical care bed numbers in Europe. Intensive Care Medicine. 2012;38(10):1647-53. https://doi. org/10.1007/s00134-012-2627-8.

5. WFSA. World Anaesthesiology Workforce. [cited 16 May 2020]. Available from https://www.wfsahq.org/workforce-map.

\section{Supplementary material}

\section{Calculation used in estimating Africa's critical care capacity}

Hospitals sampled by the African Surgical Outcomes Study had a median of 3 (IQR 0-7) critical care beds providing mechanical ventilation, with $0.9 \%$ (IQR 0-2) of hospital beds serving as critical care beds. This being the most current audit of hospital bed:critical care bed ratio in Africa, we adopted $0.9 \%$ as our conversion factor in estimating the total number of critical care beds per country. We calculated the total number of hospital beds per country from World Bank estimates (Supplementary Table I). The total number of hospital beds per country was then multiplied by $0.9 \%$ to obtain the estimated number of critical care beds. This estimate was divided by the Global Burden of Disease (GBD) 2017 country-specific population estimate to obtain critical care beds per 100000 people. Data on the number of critical care beds from the most recent national critical care capacity audits conducted in South Africa, Kenya and Uganda were used instead of estimates for these countries.

Supplementary Table I. Country-specific number and density of hospital beds, critical care beds, physicians, nurses and midwives, and anaesthesia providers

\begin{tabular}{|c|c|c|c|c|c|c|c|c|}
\hline Country & $\begin{array}{l}\text { Hospital } \\
\text { beds }\end{array}$ & $\begin{array}{c}\text { Hospital } \\
\text { beds/1 000* }\end{array}$ & $\begin{array}{l}\text { Critical care } \\
\text { beds }\end{array}$ & $\begin{array}{c}\text { Critical care } \\
\text { beds/ } \\
100000\end{array}$ & $\begin{array}{c}\text { Physicians/ } \\
1000^{*}\end{array}$ & $\begin{array}{c}\text { Nurses and } \\
\text { midwives/ } \\
1000^{*}\end{array}$ & $\begin{array}{c}\text { Total } \\
\text { anaesthesia } \\
\text { providers/ } \\
100000^{+}\end{array}$ & $\begin{array}{c}\text { Proportion of } \\
\text { non-physician } \\
\text { anaesthesia } \\
\text { providers }(\%)^{\dagger}\end{array}$ \\
\hline Algeria & 76533 & 1.9 & 689 & 1.7 & 1.8 & 2.2 & 12.73 & 69.3 \\
\hline Angola & 15640 & 0.8 & 141 & 0.5 & 0.2 & 1.3 & 0.66 & 66.7 \\
\hline Benin & 4600 & 0.5 & 41 & 0.4 & 0.2 & 0.6 & 0.70 & 78.9 \\
\hline Botswana & 3627 & 1.8 & 33 & 1.4 & 0.4 & 3.3 & 0.80 &.$\cdot$ \\
\hline Burkina Faso & 6244 & 0.4 & 56 & 0.3 & 0.1 & 0.6 & 2.77 & 89.8 \\
\hline Burundi & 7914 & 0.8 & 71 & 0.7 & 0.1 & 0.7 & 3.34 & 98.2 \\
\hline Cabo Verde & 1055 & 2.1 & 9 & 1.7 & 0.8 & 1.2 & 2.12 & 0.0 \\
\hline Cameroon & 25961 & 1.3 & 234 & 0.8 & 0.1 & 0.9 & 0.53 & 80.6 \\
\hline $\begin{array}{l}\text { Central African } \\
\text { Republic }\end{array}$ & 4476 & 1.0 & 40 & 0.9 & 0.1 & 0.2 & 0.49 & 100.0 \\
\hline Chad & 4028 & 0.4 & 36 & 0.2 & 0.0 & 0.4 & 0.14 & 95.0 \\
\hline Comoros & 1517 & 2.2 & 14 & 1.9 & 0.2 & 0.9 & .. & .. \\
\hline $\begin{array}{l}\text { Democratic Republic } \\
\text { of Congo }\end{array}$ & 45323 & 0.8 & 408 & 0.5 & 0.1 & 0.5 & 1.42 & 91.1 \\
\hline Congo & 5949 & 1.6 & 54 & 1.1 & 0.1 & 1.7 & 0.19 &.. \\
\hline Cote d'Ivoire & 7480 & 0.4 & 67 & 0.3 & 0.2 & 0.9 & 2.07 & 64.5 \\
\hline Djibouti & 1277 & 1.4 & 11 & 1.0 & 0.2 & 0.5 & 1.01 & 0.0 \\
\hline Egypt & 146896 & 1.6 & 1322 & 1.4 & 0.8 & 1.4 & 6.01 & 0.0 \\
\hline Equatorial Guinea & 1997 & 2.1 & 18 & 1.4 & 0.4 & 0.5 & .. & .. \\
\hline Eritrea & 3129 & 0.7 & 28 & 0.5 & 0.1 & 0.6 & 1.07 & 100.0 \\
\hline Eswatini & 2573 & 2.1 & 23 & 2.0 & 0.1 & 2.0 & 5.36 & 87.0 \\
\hline Ethiopia & 29961 & 0.3 & 270 & 0.3 & 0.1 & 0.8 & 0.81 & 93.6 \\
\hline Gabon & 10332 & 6.3 & 93 & 5.5 & 0.4 & 2.6 & 1.28 & 0.0 \\
\hline Gambia & 1921 & 1.1 & 17 & 0.8 & 0.1 & 1.6 & 1.96 & 89.7 \\
\hline
\end{tabular}




\begin{tabular}{|c|c|c|c|c|c|c|c|c|}
\hline Ghana & 22608 & 0.9 & 203 & 0.7 & 0.2 & 1.2 & 2.74 & 85.3 \\
\hline Guinea & 3312 & 0.3 & 30 & 0.3 & 0.1 & 0.4 & 0.34 & 93.0 \\
\hline Guinea-Bissau & 1517 & 1.0 & 14 & 0.8 & 0.2 & 1.4 & 0.11 & 0.0 \\
\hline Kenya & 57890 & 1.4 & 130 & 0.3 & 0.2 & 1.5 & 1.71 & 74.4 \\
\hline Lesotho & 2556 & 1.3 & 23 & 1.2 & 0.1 & 0.7 & 0.23 & 0.0 \\
\hline Liberia & 2930 & 0.8 & 26 & 0.6 & 0.0 & 0.1 & 1.33 & 100.0 \\
\hline Libya & 22954 & 3.7 & 207 & 3.0 & 2.2 & 6.7 & 11.23 & 53.2 \\
\hline Madagascar & 4230 & 0.2 & 38 & 0.1 & 0.2 & 0.1 & 1.05 & 78.4 \\
\hline Malawi & 20319 & 1.3 & 183 & 1.0 & 0.0 & 0.3 & 1.17 & 97.0 \\
\hline Mali & 1508 & 0.1 & 14 & 0.1 & 0.1 & 0.4 & 0.78 & 61.6 \\
\hline Mauritania & 1288 & 0.4 & 12 & 0.3 & 0.2 & 1.0 & 3.37 & 83.9 \\
\hline Mauritius & 4257 & 3.4 & 38 & 3.0 & 2.0 & 3.4 & 4.32 & 0.0 \\
\hline Morocco & 37752 & 1.1 & 340 & 1.0 & 0.7 & 1.1 & 6.25 & 69.8 \\
\hline Mozambique & 17458 & 0.7 & 157 & 0.5 & 0.1 & 0.4 & 1.13 & 81.1 \\
\hline Namibia & 5770 & 2.7 & 52 & 2.2 & 0.4 & 2.8 & 2.44 & 0.0 \\
\hline Niger & 5970 & 0.3 & 54 & 0.3 & 0.1 & 0.3 & 0.66 & 89.4 \\
\hline Nigeria & 67700 & 0.5 & 609 & 0.3 & 0.4 & 1.5 & 0.71 & 19.2 \\
\hline Rwanda & 15115 & 1.6 & 136 & 1.1 & 0.1 & 0.8 & 4.10 & 93.5 \\
\hline $\begin{array}{l}\text { Sao Tome and } \\
\text { Principe }\end{array}$ & 518 & 2.9 & 5 & 2.5 & 0.3 & 2.3 &.. &.. \\
\hline Senegal & 3660 & 0.3 & 33 & 0.2 & 0.1 & 0.3 & 0.95 & 34.7 \\
\hline Seychelles & 315 & 3.6 & 3 & 3.0 & 0.9 & 3.3 & 4.17 &.. \\
\hline Sierra Leone & 2340 & 0.4 & 21 & 0.3 & 0.0 & 1.0 & 2.05 & 98.5 \\
\hline Somalia & 12159 & 0.9 & 109 & 0.6 & 0.0 & 0.1 & 0.29 & 100.0 \\
\hline South Africa & 171818 & 2.8 & 3318 & 6.0 & 0.9 & 3.5 & 16.18 & 0.0 \\
\hline South Sudan &. &.. &.$\cdot$ & .. & 0.0 & 1.2 & 0.34 & 90.5 \\
\hline Sudan & 29480 & 0.8 & 265 & 0.7 & 0.4 & 0.8 & 0.87 & 45.7 \\
\hline Tanzania & 32270 & 0.7 & 290 & 0.5 & 0.0 & 0.4 & 0.43 & 78.3 \\
\hline Togo & 4675 & 0.7 & 42 & 0.6 & 0.0 & 0.3 & 3.01 & 92.3 \\
\hline Tunisia & 25921 & 2.3 & 233 & 2.0 & 1.3 & 2.6 & 5.06 & 0.0 \\
\hline Uganda & 16960 & 0.5 & 55 & 0.1 & 0.1 & 0.6 & 1.29 & 85.7 \\
\hline Western Sahara &.. &.. &.. & .. &.. &.. & .. &.. \\
\hline Zambia & 27700 & 2.0 & 249 & 1.4 & 0.1 & 0.9 & 0.95 & 74.7 \\
\hline Zimbabwe & 24463 & 1.7 & 220 & 1.5 & 0.0 & 1.2 & 1.79 & 68.9 \\
\hline TOTALS/ MEDIANS & 1055846 & $1(0.5-1.95)$ & 10784 & $\begin{array}{c}0.8 \\
(0.3-1.45)\end{array}$ & $\begin{array}{c}0.1 \\
(0.1-0.4)\end{array}$ & $0.9(0.5-1.5)$ & $1.28(0.7-3.01)$ & 78.65 (37.45-92) \\
\hline
\end{tabular}

*https://data.worldbank.orq/accessed on 20 March 2020; +https://www.wfsahq.org/workforce-map accessed on 20 March 2020. 